\title{
Downregulation of miRNA-15a and miRNA-16 promote tumor proliferation in multiple myeloma by increasing CABIN1 expression
}

\author{
LEI ZHANG* , LIN ZHOU*, MENG SHI, YONG KUANG and LEI FANG \\ Shi's Center of Orthopedics and Traumatology, Shuguang Hospital Affiliated to Shanghai \\ University of Traditional Chinese Medicine, Shanghai 201203, P.R. China
}

Received April 2, 2016; Accepted June 29, 2017

DOI: $10.3892 / \mathrm{ol} .2017 .7424$

\begin{abstract}
Multiple myeloma (MM) is a malignant disorder characterized by the neoplastic growth of plasma cells in the bone marrow. MicroRNAs (miRNAs/miRs) modulate key regulatory cell pathways via their influence on target genes, and may serve a crucial function in tumorigenesis. Previous studies have indicated that the downregulation of miR-15a and miR-16 contributes to MM pathogenesis. However, the functional mechanisms of miR-15a and miR-16 in MM remain unclear. In the present study, potential target sites for miR-15a and miR-16 were identified on the calcineurin-binding protein 1 (CABIN1) mRNA sequence from analyses of previously published crosslinking, ligation and sequencing of hybrids data. Again-of-function study was also performed, which determined that miR-15a/16 directly targeted CABIN1 mRNA and negatively regulated the expression of CABIN1 at the mRNA and protein level in MM cells. A cell proliferation assay demonstrated that the upregulation of miR-15a and miR-16 inhibited the proliferation of MM cells via targeting CABIN1. miR-15a and miR-16 were significantly decreased in MM specimens, compared with in normal specimens, whereas CABIN1 mRNA levels were significantly higher in MM samples compared with in normal samples. CABIN1 mRNA levels were negatively correlated with miR-15a and miR-16 expression levels in MM tissues, as determined using Pearson's correlation coefficient analysis. The results of the present study indicate that the downregulation of miR-15a and miR-16 promotes tumor
\end{abstract}

Correspondence to: Dr Yong Kuang or Dr Lei Fang, Shi's Center of Orthopedics and Traumatology, Shuguang Hospital Affiliated to Shanghai University of Traditional Chinese Medicine, 528 Zhang Heng Road, Pudong, Shanghai 201203, P.R. China

E-mail: yongk58@hotmail.com

E-mail: fangleihouse@vip.sohu.com

*Contributed equally

Key words: multiple myeloma, microRNA-15a/16, calcineurin-binding protein-1, cell proliferation proliferation in MM by increasing CABIN1 expression. The present study may aid elucidation of the functions of miR-15a and miR-16 and their function in MM carcinogenesis.

\section{Introduction}

Multiple myeloma (MM) is a malignant disorder characterized by the neoplastic growth of plasma cells in the bone marrow (1). Plasma cells are a type of white blood cell normally responsible for producing antibodies; the accumulation of abnormal plasma cells interferes with the production of normal blood cells in the bone marrow (1). MM is also characterized by the production of a paraprotein, an abnormal antibody detected in the serum or urine in the majority patients, which can cause kidney problems (2). Patients with MM exhibit a range of clinical symptoms, including bone lesions, anemia and hypercalcemia (2).

Multiple myeloma is the second most common type of hematological malignancy (comprising 13\% of these diseases), and constitutes $\sim 1 \%$ of all neoplastic diseases (2). Although strategies for the prevention, diagnosis and treatment of MM have improved, the 5-year relative survival rate is only $39 \%$ for patients with MM in Europe (3). Therefore, elucidation of the mechanisms underlying the initiation and progression of MM is essential.

MicroRNAs (miRNAs/miRs) are highly conserved short non-coding sequences of $\sim 20$ nucleotides that are able to silence target genes by acting as inhibitors of protein translation or by degrading mRNA (4). miRNAs modulate regulatory cell pathways by influencing target genes and may serve crucial functions in oncogenesis (5). Evidence indicates that miRNAs regulate MM tumorigenesis and can be valuable markers for predicting diagnosis, risk stratification and clinical outcomes (6-8).

miR-15a and miR16 are clustered at chromosomal location $13 q 14$ and possess similar sequences; they are considered to have similar tumor suppressor functions and to be involved in the regulation of cell differentiation, proliferation, apoptosis or angiogenesis in several types of human cancer, including MM (8-12). Previous studies have indicated that miR-15a/16-1 downregulation contributes to myeloma pathogenesis and mediates drug resistance in myeloma cells $(13,14)$. However, the functional mechanism of miR-15a/16 in MM remains unclear. 
In the present study, the expression of miR-15a/16 and their functional mechanism was investigated in MM cells and patients. To the best of our knowledge, the present study provides the first evidence to support miR-15a/16 directly targeting calcineurin-binding protein 1 (CABIN1) mRNA and negatively regulating them RNA and protein expression of CABIN1 in MM cells in a gain-of-function study. Using cell proliferation, the results demonstrated that the upregulation of miR-15a and miR-16 inhibited the proliferation of MM cells by targeting CABIN1. The present study also demonstrated that miR-15a and miR-16 are downregulated and negatively associated with CABIN1 mRNA levels in MM specimens. Data obtained in the present study may aid elucidation of the functions of miR-15a and miR-16 and their functions in multiple myeloma carcinogenesis.

\section{Materials and methods}

Specimens. Plasma specimens were obtained from 30 patients with MM and 10 healthy volunteers from Shuguang Hospital Affiliated to Shanghai University of Traditional Chinese Medicine (Shanghai, China). Plasma cells from patients with MM and normal controls were purified from bone marrow aspirates using CD138 MicroBeads (cat. no. 130-051-301; Miltenyi Biotec GmbH, Bergisch Gladbach, Germany), as described previously (15). All patients provided written informed consent to participate in the study. This study was approved by the Ethics Committee of Shanghai University of Traditional Chinese Medicine.

Cell culture. The U266 and RPMI-8226 MM plasma cell myeloma cell lines, and the 293 cell line, used in the present study were purchased from the American Type Culture Collection (Manassas, VA, USA). The U266 and RPMI-8226 cells were maintained in RPMI-1640 medium (Gibco; Thermo Fisher Scientific, Inc., Waltham, MA, USA) supplemented with $10 \%$ fetal bovine serum (FBS) (Thermo Fisher Scientific, Inc.). The 293 cells were cultured in Dulbecco's minimum essential medium (DMEM) (Gibco; Thermo Fisher Scientific, Inc.) containing $10 \% \mathrm{FBS}$. Cells were maintained at $37^{\circ} \mathrm{C}$ in a humidified atmosphere with $5 \% \mathrm{CO}_{2}$.

RNA isolation and reverse transcription-quantitative polymerase chain reaction $(R T-q P C R)$. Total RNA was isolated from MM tissues, adjacent non-tumor tissues and MM cell lines usingTRIzol ${ }^{\circledR}$ reagent(Invitrogen; ThermoFisherScientific,Inc.), according to the manufacturer's protocol. Purified mRNA and miRNA were detected using an RT-qPCR assay with the All-in-One miRNA RT-qPCR Detectionkit (GeneCopoeia, Inc., Rockville, MD, USA), according to the manufacturer's protocol. All primer sequences are listed in Table I. U6 small nuclear RNA was used as an internal control for the normalization and quantification of miRNA expression. $\beta$-actin was used as the internal control for the normalization and quantification of CABIN1 mRNA expression as described previously (16).

Dual-luciferase reporter assays. The luciferase reporter construct was created by cloningthehuman CABIN1 mRNA sequence into a pMIR-Report construct (Ambion; Thermo Fisher Scientific, Inc.). CABIN1 mRNA fragment (from 5178
Table I. Primers used in the present study.

\begin{tabular}{ll}
\hline Name & \multicolumn{1}{c}{ Primer sequence } \\
\hline miR-15a F & 5'-TAGCAGCACATAATGGTTTGTG-3' \\
miR-16 F & 5'-TAGCAGCACGTAAATATTGGCG-3' \\
U6 F & 5'-CTCGCTTCGGCAGCACATATAC-3' \\
U6 R & 5'-AATATGGAACGCTCACGAATTTG-3' \\
$\beta$-actin F & 5'-CAGCAAGCAGGAGTATGACG-3' \\
$\beta$-actin R & 5'-GAAAGGGTGTAACGCAACTAA-3' \\
CABIN1 F & 5'-ATCCTCACTGTGAAGGTGCTCGAA-3' \\
CABIN1 R & 5'-TTTGGTCTGCACTGTCTCCTGCAT-3' \\
CABIN1 & 5'-AAACTAGTTTGCCTGGCACATGAA \\
(reporter) F & CCG-3' \\
CABIN1 & 5'-GGAAGCTTGGTCCCGCAGCTGGGC \\
(reporter) R & CAGC-3' \\
\hline
\end{tabular}

miR-15a, microRNA-15a; F, forward primer; R, reverse primer; CABIN1, calcineurin-binding protein 1 .

to 5240) was amplified and cloned into the luciferase reporter via SpeI and HindIII sites using Ready-to-Use Seamless Cloning kit (Songon Biotech, Shanghai, China), according to the manufacturer's protocol. All primers are listed in Table I. 293 cells $\left(1 \times 10^{4}\right)$ were plated in a 96-well plate and co-transfected with $50 \mathrm{nM}$ miRNA mimics or negative control oligonucleotides RNA oligonucleotides were chemically synthesized and purified by Shanghai GenePharma Co., Ltd. (Shanghai, China). A total of $30 \mathrm{ng}$ firefly luciferase reporter and $10 \mathrm{ng}$ pRL-TK (Promega Corporation, Madison, WI, USA) were transfected into the cells using the Interferin ${ }^{\circledR}$ reagent (Polyplus-transfection SA, Illkirch, France). Cells were collected $48 \mathrm{~h}$ after the last transfection and analyzed using the Dual-Luciferase Reporter Assay system (Promega Corporation), according to the manufacturer's protocol. The firefly luciferase activity of each sample was normalized to the Renilla luciferase activity.

Oligonucleotide transfection. RNA oligonucleotides were chemically synthesized and purified by Shanghai GenePharma Co., Ltd. The sequence of human miR-15a was 5'-UAGCAG CACAUAAUGGUUUGUG-3', and that of human miR-16 was 5'-UAGCAGCACGUAAAUAUUGGCG-3'. The negative-control oligonucleotide sequence was 5'-CAGUACUUU UGUGUAGUACAA-3'. The small interfering RNA (siRNA) sequences were as follows: CABIN1 sense, 5'-CUACAUUGA GGGAACUUCATT-3' and antisense, 5'-UGAAGUUCCCUC AAUGUAGTT-3'; control sense, 5'-UUCUCCGAACGUGUC ACGUTT-3' and antisense, 5'-ACGUGACACGUUCGUAGA ATT-3'. Transfections were performed using Interferin ${ }^{\circledR}$ reagent (Polyplus-transfection SA). The final concentration of miRNA was $50 \mathrm{nM}$; the final concentration of siRNAs was $20 \mathrm{nM}$.

Cell counting kit-8 (CCK-8) assay. Cell proliferation was measured using a CCK-8 assay (Dojindo Molecular Technologies, Inc., Kumamoto, Japan), according to the manufacturer's protocol. A total of $1.0 \times 10^{4}$ cells were plated into each well of a 96-well plate and transfected with miRNA 
A

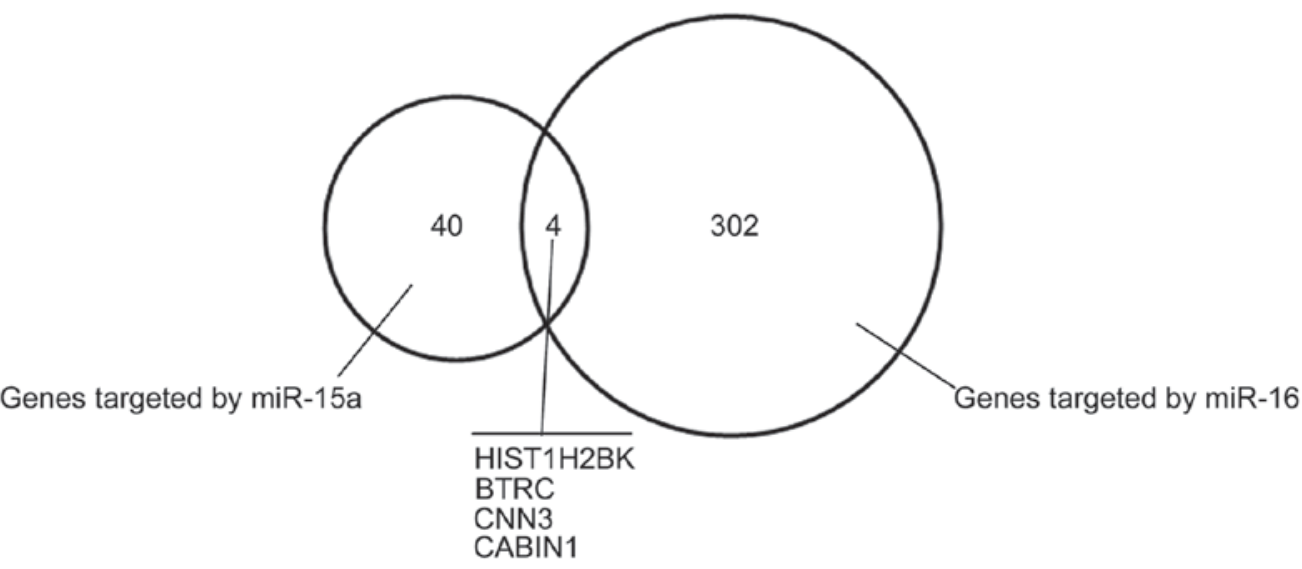

B

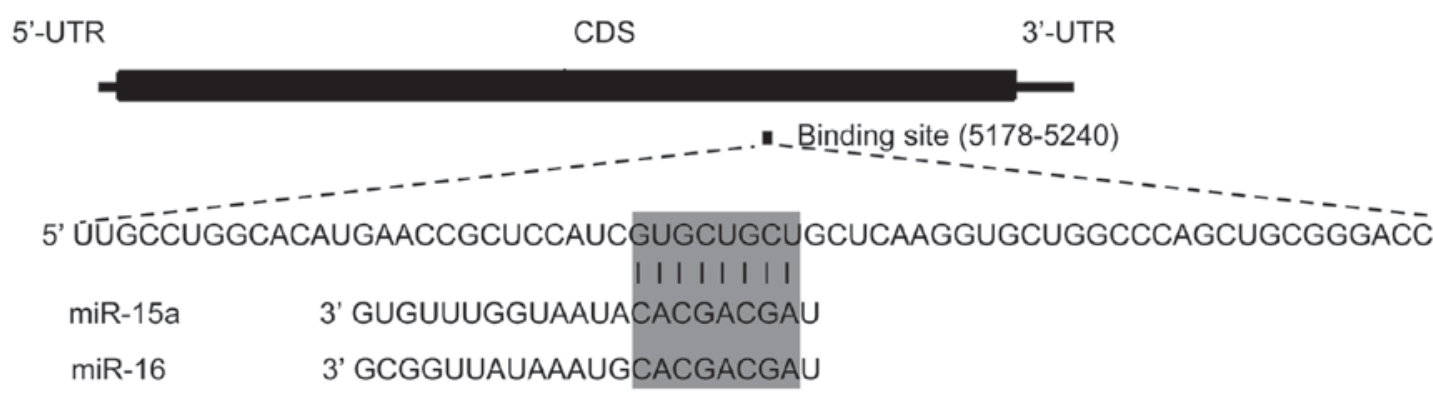

Figure 1. Bioinformatic analysis of the miR-15a/16 binding site in CABIN1 mRNA. (A) HIST1H2BK, BTRC, CNN3 and CABIN1 are targeted by miR-15a and miR-16, according to CLASH data (16). (B) Schematic diagrams of the CABIN1 mRNA and the potential binding site for the miR-15a and miR-16. The upper panel depicts a potential target site on the CDS of CABIN1; the lower panel illustrates the sequence alignment of miR-15a and miR-16 with the binding site on the CDS of CABIN1. CABIN1, calcineurin-binding protein 1, miR-15a, microRNA-15a; BTRC, $\beta$-transducin repeat containing e3 ubiquitin protein ligase; CNN3, calponin 3, UTR, untranslated region; CDS, coding sequence.

or CABIN1 siRNA to a final concentration of 50 or $20 \mathrm{nM}$, respectively using Interferin ${ }^{\circledR}$ reagent (Polyplus-transfection $\mathrm{SA}$ ), according to the manufacturer's protocol. Once the cells were harvested, $10 \mu \mathrm{l}$ CCK- 8 reagent was added to $90 \mu \mathrm{l}$ RPMI-1640 medium. The cells were subsequently incubated for $2 \mathrm{~h}$ at $37^{\circ} \mathrm{C}$ and the optical density was measured at $450 \mathrm{~nm}$. Three independent experiments were performed.

Western blot analysis. Total cellular extracts $(20 \mu \mathrm{g})$ were separated by SDS-PAGE (4-20\% Tris-glycine gel) then transferred onto a polyvinylidene fluoride membrane (Immobilon-P transfer membranes; EMD Millipore, Billerica, MA, USA). Following transfer, the blots were blocked with 5\% non-fat dry milk in PBS and $0.1 \%$ Tween-20 for $2 \mathrm{~h}$ at $4^{\circ} \mathrm{C}$ and washed three times with PBS and $0.1 \%$ Tween -20 at $4^{\circ} \mathrm{C}$. The blots were then probed with the primary anti-CABIN1 antibody (cat. no. ab3349; dilution, 1:200; Abcam, Cambridge, MA, USA), CDKN1A (cat. no. sc-6246; dilution, 1:200) and $\beta$-actin (cat.no. c-11;1: 200; Santa Cruz Biotechnology, Inc.). The blots were then probed with the horseradish peroxidase-conjugated goat anti-rabbit IgG H\&L secondary antibody, (cat. no. ab6721, dilution, 1:750; Abcam) for $1 \mathrm{~h}$ at $4^{\circ} \mathrm{C}$, followed by washes in PBS + 0.1\% Tween-20, and then detected using an Odyssey Scanning system (Gene Company, Ltd., Hong Kong, China).

Statistical analysis. All statistical analyses were performed using the SPSS 16.0 statistical software package (SPSS,
Inc., Chicago, IL, USA). Quantitative data is expressed as mean \pm standard deviation. Data of $m R N A$ expression levels in MM specimens compared to healthy volunteers were subjected to Student's t-test analysis. Data from dual-luciferase reporter assays and CCK-8 assay were analyzed by one-way analysis of variance followed by the Student-Newman-Keuls post-test. $\mathrm{P}<0.05$ was considered to indicate a statistically significant difference. Pearson's correlation coefficient and Spearman's correlation coefficient was used forthe comparison of mRNA expression of miRNA and its target gene.

\section{Results}

miR-15a/16 have potential target sites on CABIN1 mRNA. miR-15a and miR-16, whose expression is decreased or absent in MM, have similar sequences and are considered to have the same target genes. To investigate the biological significance of these miRNAs and the underlying mechanisms of their downregulation in MM, the potential target genes of miR-15a/16 that have functions in MM pathogenesis were further analyzed.

Previously published crosslinking, ligation, and sequencing of hybrids (CLASH) data in 293 cells provided direct evidence for the miRNA-mRNA pairing (17). The CLASH data revealed that four genes, HIST1H2K, BTRC, CNN3 and CABIN1, aretargeted by miR-15a and miR-16 (Fig. 1A). All genes targeted by miR-15aor miR-16 in the CLASH dataset (17) are listed in Table II. CABIN1 is involved in several cellular 
Table II. List of genes that paired with miR-15a and miR-16 in CLASH data.

miRNA Target gene

miR-15a ENSG00000099991_ENST00000398319_CABIN1

miR-16 ENSG00000099991_ENST00000398319_CABIN1

miR-15a ENSG00000110429_ENST00000265651_FBXO3

miR-16 ENSG00000110429_ENST00000265651_FBXO3

miR-15a ENSG00000117519_ENST00000370206_CNN3

miR-16 ENSG00000117519_ENST00000370206_CNN3

miR-15a ENSG00000197903_ENST00000396891_HIST1H2BK

miR-16 ENSG00000197903_ENST00000396891_HIST1H2BK

miR-15a ENSG00000013275_ENST00000157812_PSMC4

miR-15a ENSG00000015568_ENST00000016946_RGPD5

miR-15a ENSG00000023909_ENST00000370238_GCLM

miR-15a ENSG00000025796_ENST00000369002_SEC63

miR-15a ENSG00000057608_ENST00000380191_GDI2

miR-15a ENSG00000069956_ENST00000261845_MAPK6

miR-15a ENSG00000071553_ENST00000369762_ATP6AP1

miR-15a ENSG00000073009_ENST00000369606_IKBKG

miR-15a ENSG00000110092_ENST00000227507_CCND1

miR-15a ENSG00000110435_ENST00000227868_PDHX

miR-15a ENSG00000111641_ENST00000399466_NOP2

miR-15a ENSG00000111642_ENST00000357008_CHD4

miR-15a ENSG00000113312_ENST00000231238_TTC1

miR-15a ENSG00000115758_ENST00000234111_ODC1

miR-15a ENSG00000115966_ENST00000264110_ATF2

miR-15a ENSG00000117215_ENST00000375105_PLA2G2D

miR-15a ENSG00000118971_ENST00000261254_CCND2

miR-15a ENSG00000119777_ENST00000238788_TMEM214

miR-15a ENSG00000121940_ENST00000415331_CLCC1

miR-15aＥNSG00000122786_ENST00000361901_CALD1

miR-15a ENSG00000129515_ENST00000396526_SNX6

miR-15a ENSG00000130294_ENST00000320389_KIF1A

miR-15a ENSG00000130726_ENST00000253024_TRIM28

miR-15a ENSG00000131023_ENST00000253339_BX276089-1

miR-15a ENSG00000132141_ENST00000314144_CCT6B

miR-15a ENSG00000133059_ENST00000367162_DSTYK

miR-15a ENSG00000135503_ENST00000257963_ACVR1B

miR-15a ENSG00000138073_ENST00000260643_PREB

miR-15a ENSG00000138381_ENST00000260952_ASNSD1

miR-15a ENSG00000138386_ENST00000337386_NAB1

miR-15a ENSG00000148688_ENST00000413330_RPP30

miR-15a ENSG00000150907_ENST00000379561_FOXO1

miR-15a ENSG00000163249_ENST00000295414_CCNYL1

miR-15a ENSG00000165494_ENST00000298281_PCF11

miR-15a ENSG00000165672_ENST00000298510_PRDX3

miR-15a ENSG00000166167_ENST00000370187_BTRC

miR-15a ENSG00000169710_ENST00000306749_FASN

miR-15a ENSG00000185658_ENST00000341322_BRWD1

miR-15a ENSG00000185697_ENST00000402282_MYBL1

miR-15a ENSG00000188647_ENST00000415701_PTAR1

miR-16 ENSG00000005302_ENST00000337339_MSL3

miR-16 ENSG00000005700_ENST00000306270_IBTK

miR-16 ENSG00000008438_ENST00000008938_PGLYRP1
Table II. Continued.

miRNA Target gene

miR-16 ENSG00000008516_ENST00000336577_MMP25

miR-16 ENSG00000008838_ENST00000394126_MED24

miR-16 ENSG00000009954_ENST00000339594_BAZ1B

miR-16 ENSG00000010322_ENST00000345716_NISCH

miR-16 ENSG00000010404_ENST00000340855_IDS

miR-16 ENSG00000011028_ENST00000303375_MRC2

miR-16 ENSG00000035403_ENST00000372755_VCL

miR-16 ENSG00000037474_ENST00000264670_NSUN2

miR-16 ENSG00000050405_ENST00000341247_LIMA1

miR-16 ENSG00000056277_ENST00000370978_ZNF280C

miR-16 ENSG00000063046_ENST00000262056_EIF4B

miR-16 ENSG00000064419_ENST00000265388_TNPO3

miR-16 ENSG00000065150_ENST00000261574_IPO5

miR-16 ENSG00000065526_ENST00000375759_SPEN

miR-16 ENSG00000065665_ENST00000379033_SEC61A2

miR-16 ENSG00000066926_ENST00000262093_FECH

miR-16 ENSG00000067836_ENST00000322048_ROGDI

miR-16 ENSG00000069998_ENST00000336737_CECR5

miR-16 ENSG00000070501_ENST00000265421_POLB

miR-16 ENSG00000070882_ENST00000313367_OSBPL3

miR-16 ENSG00000072110_ENST00000193403_ACTN1

miR-16 ENSG00000072135_ENST00000175756_PTPN18

miR-16 ENSG00000072274_ENST00000360110_TFRC

miR-16 ENSG00000072803_ENST00000296933_FBXW11

miR-16 ENSG00000074755_ENST00000381638_ZZEF1

miR-16 ENSG00000075292_ENST00000409544_ZNF638

miR-16 ENSG00000075624_ENST00000331789_ACTB

miR-16 ENSG00000076604_ENST00000262395_TRAF4

miR-16 ENSG00000076826_ENST00000446248_KIAA1543

miR-16 ENSG00000083544_ENST00000411610_TDRD3

miR-16 ENSG00000085872_ENST00000198939_CHERP

miR-16 ENSG00000086570_ENST00000261800_FAT2

miR-16 ENSG00000086712_ENST00000380122_CXorf15

miR-16 ENSG00000088387_ENST00000357329_DOCK9

miR-16 ENSG00000088899_ENST00000329152_

RP5-1187M17-10

miR-16 ENSG00000089157_ENST00000228306_RPLP0

miR-16 ENSG00000089693_ENST00000203630_MLF2

miR-16 ENSG00000090376_ENST00000457197_IRAK3

miR-16 ENSG00000090621_ENST00000372857_PABPC4

miR-16 ENSG00000091164_ENST00000217515_TXNL1

miR-16 ENSG00000093000_ENST00000347635_NUP50

miR-16 ENSG00000093010_ENST00000361682_COMT

miR-16 ENSG00000095564_ENST00000265990_BTAF1

miR-16 ENSG00000095739_ENST00000375533_BAMBI

miR-16 ENSG00000096968_ENST00000381652_JAK2

miR-16 ENSG00000099203_ENST00000214869_TMED1

miR-16 ENSG00000099991_ENST00000398319_CABIN1

miR-16 ENSG00000100234_ENST00000266085_TIMP3

miR-16 ENSG00000100241_ENST00000380817_SBF1

miR-16 ENSG00000100304_ENST00000216129_TTLL12

miR-16 ENSG00000100304_ENST00000216129_TTLL12 
Table II. Continued.

miRNA Target gene

miR-16 ENSG00000100697_ENST00000343455_DICER1

miR-16 ENSG00000100764_ENST00000261303_PSMC1

miR-16 ENSG00000101166_ENST00000355937_SLMO2

miR-16 ENSG00000101190_ENST00000335351_TCFL5

miR-16 ENSG00000101294_ENST00000340852_HM13

miR-16 ENSG00000101452_ENST00000252011_DHX35

miR-16 ENSG00000101782_ENST00000339486_RIOK3

miR-16 ENSG00000102174_ENST00000379374_PHEX

miR-16 ENSG00000102931_ENST00000219204_ARL2BP

miR-16 ENSG00000103148_ENST00000399953_C16orf35

miR-16 ENSG00000103222_ENST00000399410_ABCC1

miR-16 ENSG00000103319_ENST00000263026_EEF2K

miR-16 ENSG00000103489_ENST00000261381_XYLT1

miR-16 ENSG00000104331_ENST00000262644_IMPAD1

miR-16 ENSG00000104341_ENST00000445593_LAPTM4B

miR-16 ENSG00000104824_ENST00000221419_HNRNPL

miR-16 ENSG00000104824_ENST00000221419_HNRNPL

miR-16 ENSG00000105173_ENST00000262643_CCNE1

miR-16 ENSG00000105223_ENST00000409587_PLD3

miR-16 ENSG00000105281_ENST00000306894_SLC1A5

miR-16 ENSG00000105429_ENST00000334370_MEGF8

miR-16 ENSG00000105784_ENST00000338056_RUNDC3B

miR-16 ENSG00000106009_ENST00000340611_C7orf27

miR-16 ENSG00000106355_ENST00000450169_LSM5

miR-16 ENSG00000106665_ENST00000361545_CLIP2

miR-16 ENSG00000107937_ENST00000360803_GTPBP4

miR-16 ENSG00000108510_ENST00000397786_MED13

miR-16 ENSG00000109775_ENST00000264689_UFSP2

miR-16 ENSG00000109971_ENST00000227378_HSPA8

miR-16 ENSG00000109971_ENST00000227378_HSPA8

miR-16 ENSG00000110367_ENST00000264018_DDX6

miR-16 ENSG00000110713_ENST00000324932_NUP98

miR-16 ENSG00000111012_ENST00000228606_CYP27B1

miR-16 ENSG00000112242_ENST00000346618_E2F3

miR-16 ENSG00000112306_ENST00000230050_RPS12

miR-16 ENSG00000112592_ENST00000392092_TBP

miR-16 ENSG00000112664_ENST00000358797_NUDT3

miR-16 ENSG00000112701_ENST00000447266_SENP6

miR-16 ENSG00000113569_ENST00000231498_NUP155

miR-16 ENSG00000113732_ENST00000519374_ATP6V0E1

miR-16 ENSG00000114030_ENST00000344337_KPNA1

miR-16 ENSG00000114933_ENST00000403263_INO80D

miR-16 ENSG00000114982_ENST00000354204_KIAA1310

miR-16 ENSG00000115307_ENST00000377526_AUP1

miR-16 ENSG00000115616_ENST00000233969_SLC9A2

miR-16 ENSG00000116396_ENST00000369787_KCNC4

miR-16 ENSG00000116685_ENST00000376572_KIAA2013

miR-16 ENSG00000116748_ENST00000369538_AMPD1

miR-16 ENSG00000116786_ENST00000420314_PLEKHM2

miR-16 ENSG00000117984_ENST00000236671_CTSD

miR-16 ENSG00000120158_ENST00000381750_RCL1

miR-16 ENSG00000120438_ENST00000321394_TCP1
Table II. Continued.

miRNA Target gene

miR-16 ENSG00000120833_ENST00000340600_SOCS2

miR-16 ENSG00000122482_ENST00000370440_ZNF644

miR-16 ENSG00000122566_ENST00000354667_ HNRNPA2B 1

miR-16 ENSG00000123384_ENST00000243077_AC137834-1

miR-16 ENSG00000123411_ENST00000262032_IKZF4

miR-16 ENSG00000124529_ENST00000377364_HIST1H4B

miR-16 ENSG00000125166_ENST00000245206_GOT2

miR-16 ENSG00000125166_ENST00000245206_GOT2

miR-16 ENSG00000126016_ENST00000304758_AMOT

miR-16 ENSG00000126464_ENST00000418929_PRR12

miR-16 ENSG00000126602_ENST00000246957_TRAP1

miR-16 ENSG00000127152_ENST00000345514_BCL11B

miR-16 ENSG00000127616_ENST00000429416_SMARCA4

miR-16 ENSG00000128714_ENST00000392539_HOXD13

miR-16 ENSG00000129566_ENST00000262715_TEP1

miR-16 ENSG00000130054_ENST00000252338_FAM155B

miR-16 ENSG00000130227_ENST00000252512_XPO7

miR-16 ENSG00000130402_ENST00000252699_ACTN4

miR-16 ENSG00000130640_ENST00000368563_TUBGCP2

miR-16 ENSG00000130733_ENST00000393508_YIPF2

miR-16 ENSG00000131269_ENST00000253577_ABCB7

miR-16 ENSG00000131467_ENST00000441946_PSME3

miR-16 ENSG00000132153_ENST00000443212_DHX30

miR-16 ENSG00000132341_ENST00000448750_RAN

miR-16 ENSG00000132423_ENST00000254759_COQ3

miR-16 ENSG00000132676_ENST00000368336_DAP3

miR-16 ENSG00000132718_ENST00000368324_SYT11

miR-16 ENSG00000132953_ENST00000255305_XPO4

miR-16 ENSG00000133124_ENST00000372129_IRS4

miR-16 ENSG00000133243_ENST00000255608_BTBD2

miR-16 ENSG00000133433_ENST00000290765_GSTT2B

miR-16 ENSG00000133773_ENST00000256151_CCDC59

miR-16 ENSG00000134049_ENST00000256433_IER3IP1

miR-16 ENSG00000134817_ENST00000257254_APLNR

miR-16 ENSG00000134871_ENST00000360467_COL4A2

miR-16 ENSG00000134970_ENST00000456936_TMED7

miR-16 ENSG00000135269_ENST00000358204_TES

miR-16 ENSG00000135341_ENST00000369329_MAP3K7

miR-16 ENSG00000135898_ENST00000392040_GPR55

miR-16 ENSG00000136695_ENST00000393200_IL1F5

miR-16 ENSG00000136827_ENST00000437532_TOR1A

miR-16 ENSG00000137713_ENST00000427203_PPP2R1B

miR-16 ENSG00000138041_ENST00000272313_SMEK2

miR-16 ENSG00000138326_ENST00000435275_RPS24

miR-16 ENSG00000138326_ENST00000435275_RPS24

miR-16 ENSG00000138399_ENST00000453153_FASTKD1

miR-16 ENSG00000138592_ENST00000433963_USP8

miR-16 ENSG00000138802_ENST00000265175_SEC24B

miR-16 ENSG00000139117_ENST00000331366_CPNE8

miR-16 ENSG00000140391_ENST00000267970_TSPAN3

miR-16 ENSG00000140526_ENST00000355100_ABHD2 
Table II. Continued.

\begin{tabular}{ll}
\hline miRNA & Target gene \\
\hline
\end{tabular}

miR-16 ENSG00000140829_ENST00000268482_DHX38

miR-16 ENSG00000140955_ENST00000315906_ADAD2

miR-16 ENSG00000141101_ENST00000268802_NOB1

miR-16 ENSG00000141140_ENST00000378326_MYO19

miR-16 ENSG00000141367_ENST00000269122_CLTC

miR-16 ENSG00000141367_ENST00000269122_CLTC

miR-16 ENSG00000141404_ENST00000334049_GNAL

miR-16 ENSG00000141720_ENST00000269554_PIP5K2B

miR-16 ENSG00000142235_ENST00000270238_LMTK3

miR-16 ENSG00000142961_ENST00000371940_MOBKL2C

miR-16 ENSG00000144227_ENST00000272641_NXPH2

miR-16 ENSG00000144659_ENST00000273158_SLC25A38

miR-16 ENSG00000144852_ENST00000393716_NR1I2

miR-16 ENSG00000145495_ENST00000274140_MARCH6

miR-16 ENSG00000145495_ENST00000274140_MARCH6

miR-16 ENSG00000146833_ENST00000349062_TRIM4

miR-16 ENSG00000147255_ENST00000370904_IGSF1

miR-16 ENSG00000147403_ENST00000424325_RPL10

miR-16 ENSG00000147408_ENST00000454498_

CSGALNACT1

miR-16 ENSG00000147533_ENST00000520817_GOLGA7

miR-16 ENSG00000149273_ENST00000278572_RPS3

miR-16 ENSG00000150764_ENST00000440460_DIXDC1

miR-16 ENSG00000151612_ENST00000379448_ZNF827

miR-16 ENSG00000152234_ENST00000282050_ATP5A1

miR-16 ENSG00000152270_ENST00000282096_PDE3B

miR-16 ENSG00000152291_ENST00000377386_TGOLN2

miR-16 ENSG00000154124_ENST00000284274_FAM105B

miR-16 ENSG00000154760_ENST00000285013_SLFN13

miR-16 ENSG00000155393_ENST00000299192_HEATR3

miR-16 ENSG00000156110_ENST00000372734_ADK

miR-16 ENSG00000156261_ENST00000286788_CCT8

miR-16 ENSG00000156304_ENST00000286835_SFRS15

miR-16 ENSG00000156486_ENST00000521839_KCNS2

miR-16 ENSG00000156508_ENST00000316292_EEF1A1

miR-16 ENSG00000159202_ENST00000360943_UBE2Z

miR-16 ENSG00000159348_ENST00000367249_CYB5R1

miR-16 ENSG00000160209_ENST00000291565_PDXK

miR-16 ENSG00000160767_ENST00000361361_FAM189B

miR-16 ENSG00000160991_ENST00000356387_ORAI2

miR-16 ENSG00000161542_ENST00000446526_PRPSAP1

miR-16 ENSG00000162300_ENST00000294258_ZFPL1

miR-16 ENSG00000163013_ENST00000521871_FBXO41

miR-16 ENSG00000163113_ENST00000369135_OTUD7B

miR-16 ENSG00000163527_ENST00000295770_STT3B

miR-16 ENSG00000163946_ENST00000431842_C3orf63

miR-16 ENSG00000164076_ENST00000477224_CAMKV

miR-16 ENSG00000164300_ENST00000507668_SERINC5

miR-16 ENSG00000164818_ENST00000297440_HEATR2

miR-16 ENSG00000164889_ENST00000485713_SLC4A2

miR-16 ENSG00000165629_ENST00000356708_ATP5C1

miR-16 ENSG00000165995_ENST00000377315_CACNB2
Table II. Continued.

miRNA Target gene

miR-16 ENSG00000165995_ENST00000396576_CACNB2 miR-16 ENSG00000166105_ENST00000431683_GLB1L3 miR-16 ENSG00000166167_ENST00000370187_BTRC miR-16 ENSG00000166681_ENST00000372645_NGFRAP1 miR-16 ENSG00000166833_ENST00000396085_NAV2 miR-16 ENSG00000167397_ENST00000394975_VKORC1 miR-16 ENSG00000167552_ENST00000301071_TUBA1A miR-16 ENSG00000167721_ENST00000301364_TSR1 miR-16 ENSG00000167842_ENST00000381165_MIS12 miR-16 ENSG00000168137_ENST00000402198_SETD5 miR-16 ENSG00000168268_ENST00000422318_NT5DC2 miR-16 ENSG00000168488_ENST00000336783_ATXN2L miR-16 ENSG00000168758_ENST00000305476_SEMA4C miR-16 ENSG00000169180_ENST00000304658_XPO6 miR-16 ENSG00000169251_ENST00000351193_NMD3 miR-16 ENSG00000169813_ENST00000443950_HNRNPF miR-16 ENSG00000170004_ENST00000380358_CHD3 miR-16 ENSG00000170006_ENST00000304385_TMEM154 miR-16 ENSG00000170500_ENST00000393437_LONRF2 miR-16 ENSG00000170759_ENST00000302418_KIF5B miR-16 ENSG00000170776_ENST00000394518_AKAP13 miR-16 ENSG00000171150_ENST00000306503_SOCS5 miR-16 ENSG00000171385_ENST00000369697_KCND3 miR-16 ENSG00000171490_ENST00000396503_RSL1D1 miR-16 ENSG00000171570_ENST00000406058_EGLN2 miR-16 ENSG00000172354_ENST00000393926_GNB2 miR-16 ENSG00000173068_ENST00000380672_BNC2 miR-16 ENSG00000173638_ENST00000311124_SLC19A1 miR-16 ENSG00000173786_ENST00000393892_CNP miR-16 ENSG00000173825_ENST00000309880_TIGD3 miR-16 ENSG00000173875_ENST00000343325_ZNF791 miR-16 ENSG00000173915_ENST00000337003_USMG5 miR-16 ENSG00000174231_ENST00000304992_PRPF8 miR-16 ENSG00000174292_ENST00000311668_TNK1 miR-16 ENSG00000174405_ENST00000356922_LIG4 miR-16 ENSG00000174595_ENST00000310992_KLF14 miR-16 ENSG00000175414_ENST00000310389_ARL10 miR-16 ENSG00000175467_ENST00000312397_SART1 miR-16 ENSG00000177169_ENST00000321867_ULK1 miR-16 ENSG00000179915_ENST00000406316_NRXN1 miR-16 ENSG00000180596_ENST00000396984_HIST1H2BC miR-16 ENSG00000180758_ENST00000377411_GPR157 miR-16 ENSG00000181031_ENST00000323434_RPH3AL miR-16 ENSG00000181192_ENST00000263035_DHTKD1 miR-16 ENSG00000181222_ENST00000322644_POLR2A miR-16 ENSG00000181418_ENST00000421952_DDN miR-16 ENSG00000181472_ENST00000325144_ZBTB2 miR-16 ENSG00000182149_ENST00000329908_KIAA0174 miR-16 ENSG00000182158_ENST00000330387_CREB3L2 miR-16 ENSG00000182447_ENST00000327928_OTOL1 miR-16 ENSG00000182611_ENST00000333151_HIST1H2AJ miR-16 ENSG00000183638_ENST00000382483_RP1L1 
Table II. Continued.

miRNA Target gene

miR-16 ENSG00000184009_ENST00000331925_ACTG1

miR-16 ENSG00000184436_ENST00000215742_THAP7

miR-16 ENSG00000184634_ENST00000374080_MED12

miR-16 ENSG00000184675_ENST00000330258_FAM123B

miR-16 ENSG00000184779_ENST00000330339_RPS17

miR-16 ENSG00000184867_ENST00000328766_ARMCX2

miR-16 ENSG00000185176_ENST00000407834_AQP12B

miR-16 ENSG00000185818_ENST00000331662_NAT8L

miR-16 ENSG00000185915_ENST00000379499_KLHL34

miR-16 ENSG00000186765_ENST00000334850_FSCN2

miR-16 ENSG00000187239_ENST00000446176_FNBP1

miR-16 ENSG00000187498_ENST00000375820_COL4A1

miR-16 ENSG00000187583_ENST00000379410_PLEKHN1

miR-16 ENSG00000187837_ENST00000343677_HIST1H1C

miR-16 ENSG00000187837_ENST00000343677_HIST1H1C

miR-16 ENSG00000188486_ENST00000375167_H2AFX

miR-16 ENSG00000188493_ENST00000378313_C19orf54

miR-16 ENSG00000189091_ENST00000302516_SF3B3

miR-16 ENSG00000189292_ENST00000403610_FAM150B

miR-16 ENSG00000189334_ENST00000368702_S100A14

miR-16 ENSG00000196090_ENST00000373198_PTPRT

miR-16 ENSG00000196235_ENST00000378524_SUPT5H

miR-16 ENSG00000196365_ENST00000360614_LONP1

miR-16 ENSG00000196604_ENST00000357462_A26C1B

miR-16 ENSG00000196924_ENST00000369863_FLNA

miR-16 ENSG00000196981_ENST00000330689_WDR5B

miR-16 ENSG00000197157_ENST00000354725_SND1

miR-16 ENSG00000197353_ENST00000359228_LYPD2

miR-16 ENSG00000197386_ENST00000355072_HTT

miR-16 ENSG00000197858_ENST00000355091_GPAA1

miR-16 ENSG00000198586_ENST00000431350_TLK1

miR-16 ENSG00000198804_ENST00000361624_MT-CO1

miR-16 ENSG00000205581_ENST00000380749_HMGN1

miR-16 ENSG00000205707_ENST00000381356_LYRM5

miR-16 ENSG00000215041_ENST00000399464_NEURL4

miR-16 ENSG00000215114_ENST00000399598_UBXN2B

miR-16 ENSG00000231555_ENST00000445736_HSPA1B

miR-16 ENSG00000232804_ENST00000450744_HSPA1B

miR-16 ENSG00000235067_ENST00000419792_TUBB

miR-16 ENSG00000237405_ENST00000447921_AGER

miR-16 ENSG00000244476_ENST00000472091_RP4-761I2-4

miR-16 ENSG00000253953_ENST00000519479_PCDHGB4

miR-15a, microRNA-15a.

processes including cell proliferation and the restraint of p53 activity on chromatin (18); therefore, CABIN1 was selected for further investigation. Alignment results indicated that the potential targeting sequence for $\mathrm{miR}-15 \mathrm{a} / 16$ was within the protein-coding region of CABIN1 mRNA between nucleotides 5,178 and 5,240 (Fig. 1B).
miR-15a/16 directly target and downregulate CABIN1 expression in MM cells. Previous studies have reported that the expression of miR-15 and miR-16 was downregulated in MM tissues $(8,19)$. To determine whether miR-15a and miR-16 are capable of targeting and regulating CABIN1 expression in $\mathrm{MM}$, a gain-of-function study was performed.

Luciferase reporter plasmids were created with targeting sequences of CABIN1 mRNA (Fig. 2A). miR-15a and miR-16 were transfected into 293 cells and a dual-luciferase reporter assay was used to assess the miRNA regulation of CABIN1. The results revealed that the upregulation of miR-15a or miR-16 decreased luciferase activity, whereas a reporter plasmid without the presence ofCABIN1 sequence produced no change in luciferase activity (Fig. 2B). These results indicated miR-15a and miR-16 negatively regulated CABIN1 expression.

Whether the endogenous CABIN1 in MM cells was similarly regulated was subsequently investigated. The MMU266 and RPMI-8226 cells lines were transfected with miR-15a and miR-16, and CABIN1 mRNA and protein levels were examined by RT-qPCR and western blotting, respectively. The levels of CABIN1 mRNA (Fig. 2C) and protein (Fig. 2D) were consistently and substantially downregulated by miR-15a and miR-16 in MM cells. The results of the present study indicated that miR-15a and miR-16 directly targeted CABIN1 mRNA and negatively regulated expression of CABIN1 at the mRNA and protein level.

Upregulation of miR-15a and miR-16 inhibits the proliferation of MM cells by targeting CABIN1. Overexpression of CABIN1 increased the proliferation of cancer cells by physically interacting with p53 and repressing p53 transcriptional activity on target genes, including CDKN1A (18). Given that CABIN1 is a direct target of miR-15a/16, it was hypothesized that miR-15a and miR-16 may affect MM cell viability through CABIN1. miR-15aand miR-16a or CABIN1 siRNA was transiently transfected into U266 and RPMI-8226 cells. The results of western blotting revealed that transfection with miR-15a and miR-16or CABIN1-targeted siRNA decreased the expression of CABIN1 and p53 downstream gene CDKN1A in U266 and RPMI-8226 cells (Fig. 3A).

Using a cell proliferation assay, the overexpression of miR-15a and miR-16 in U266 and RPMI-8226 cells was determined to result in the significant suppression of cell proliferation, similar to CABIN1 siRNA (Fig. 3B). The results of the present study demonstrated that the downregulation of CABIN1 expression by miR-15a and miR-16 contributes, partially, to the suppression of MM cell growth.

miR-15a and miR-16 are downregulated and negatively associated with CABIN1 mRNA levels in MM specimens. Previous studies have reported that miR-15a and miR-16 downregulated in several types of cancer, including MM $(8,19)$. Overexpression of CABIN1 is associated with poorer patient outcomes in a number of cancer types $(18,20,21)$. The results of the present study demonstrated that CABIN1 is negatively regulated by miR-15a and miR-16 in MM cells. Next, the correlation between miR-15a and miR-16 expression levels and CABIN1 mRNA levels was assessed in MM specimens.

To determine the levels of miR-15a and miR-16 in MM samples, total RNA was extracted from MM specimens 
A

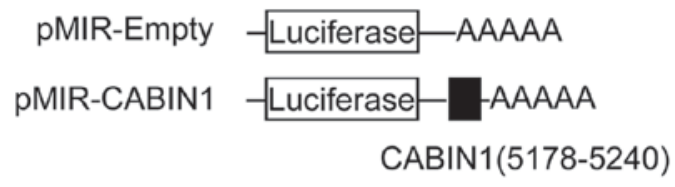

B

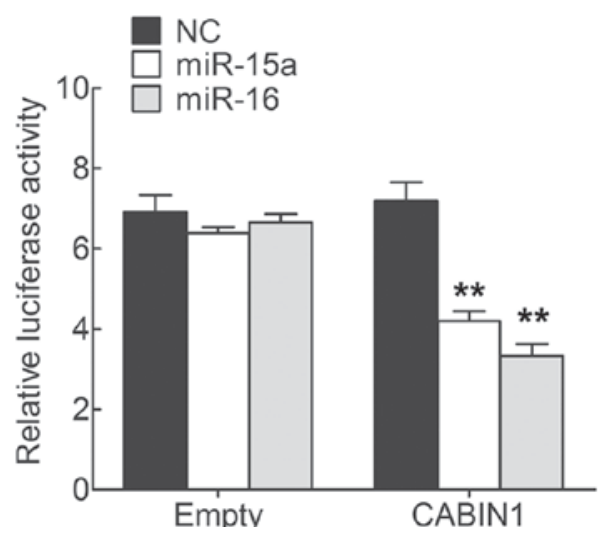

D

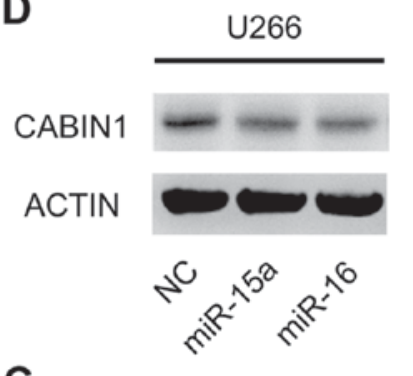

C

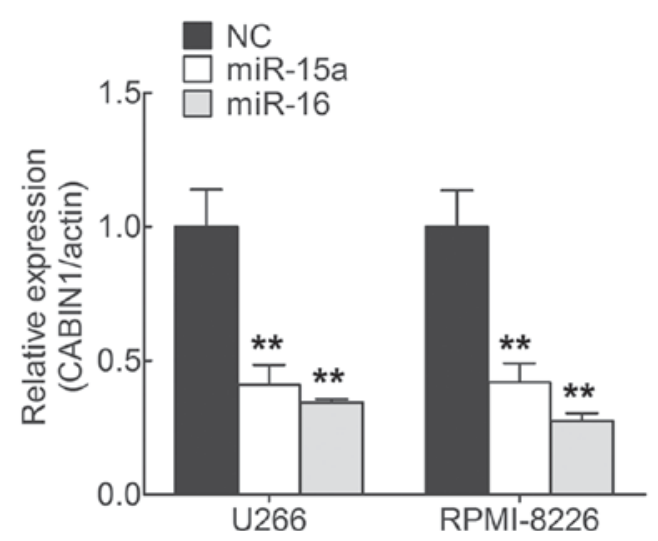

Figure 2. miR-15a and miR-16 target CABIN1 in MM cells. (A) Schematic representation of pMIR firefly luciferase reporter construction. The potential binding site of miR-15a and miR-16 is indicated in Fig. 1B. (B) Analysis of luciferase activity in 293 cells. Cells were co-transfected with a firefly luciferase reporter plasmid containing putative miR-15a- and miR-16-targeting sequences; $48 \mathrm{~h}$ after transfection, cell lysates were assayed for luciferase activity and normalized to Renilla luciferase activity. (C and D) Effects of miR-15a and miR-16 on the endogenous expression level of CABIN1. U266 and RPMI-8226 MM cells were co-transfected with miR-15a or miR-16 and negative control oligonucleotides and $48 \mathrm{~h}$ after transfection, cells were isolated. The (C) mRNA levels and (D) protein levels of CABIN1 were analyzed by RT-qPCR and western blot analysis, respectively. ${ }^{* *} \mathrm{P}<0.01$ by one-way analysis of variance followed by the Student Newman-Keuls test. miR-15a, microRNA-15a; CANIB1, calcineurin-binding protein 3; MM, multiple myeloma; NC, negative control.

A
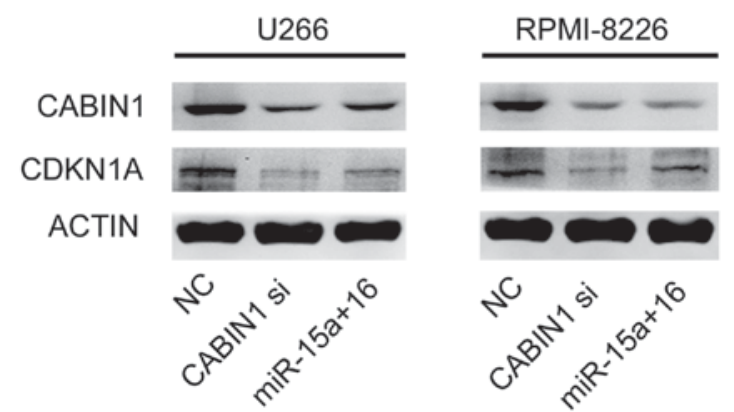

B
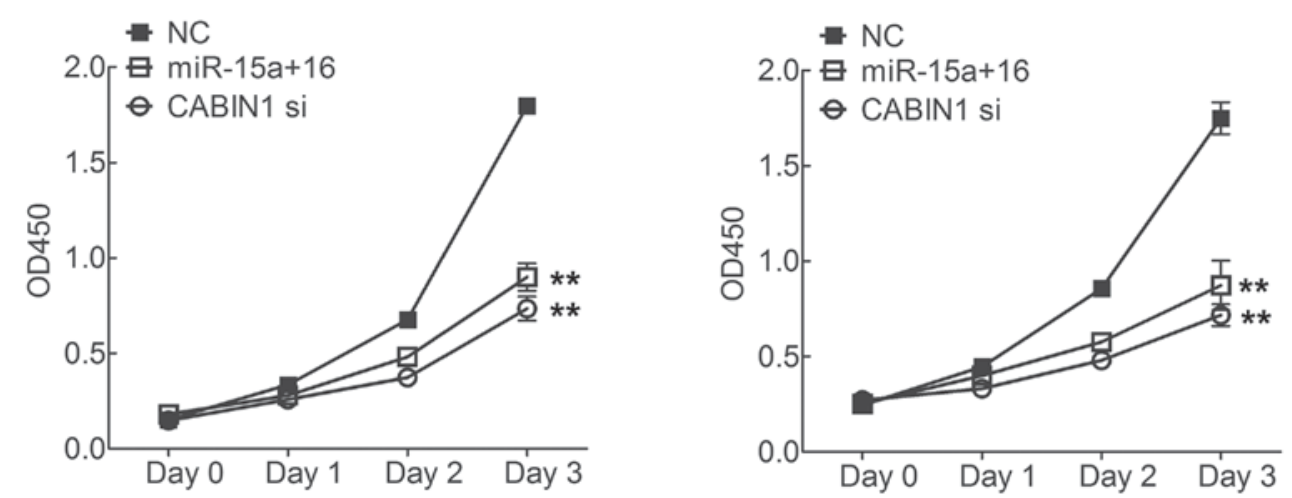

Figure 3. Upregulation of miR-15a and miR-16 inhibits proliferation of MM cells through targeting of CABIN1. (A) Western blot analysis was performed to determine the expression level of CABIN1 and CDKN1A following transfection of CABIN1 siRNA or miR-15a and miR-16 in U266 and RPMI-8226 cells. (B) The effects of CABIN1 siRNA or miR-15a and miR-16 transfection on U266 and RPMI-8226 cell proliferation were determined using a Cell Counting kit- 8 assay at the indicated times following transfection. ${ }^{* *} \mathrm{P}<0.01$ by one-way analysis of variance followed by the Student Newman-Keuls test. miR-15a, microRNA-15a; CANIB1, calcineurin-binding protein 1; CDKN1A, cyclin-dependent kinase inhibitor 1 $\alpha$; siRNA, small interfering RNA. 
A

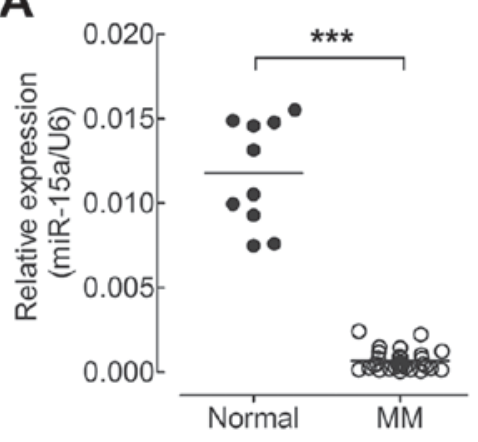

C

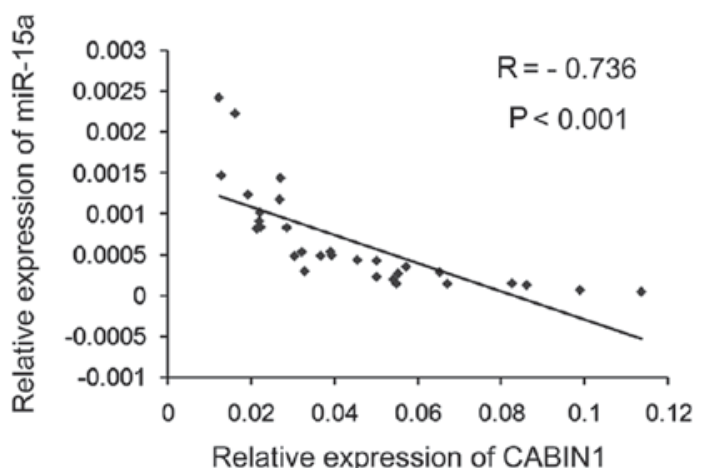

B
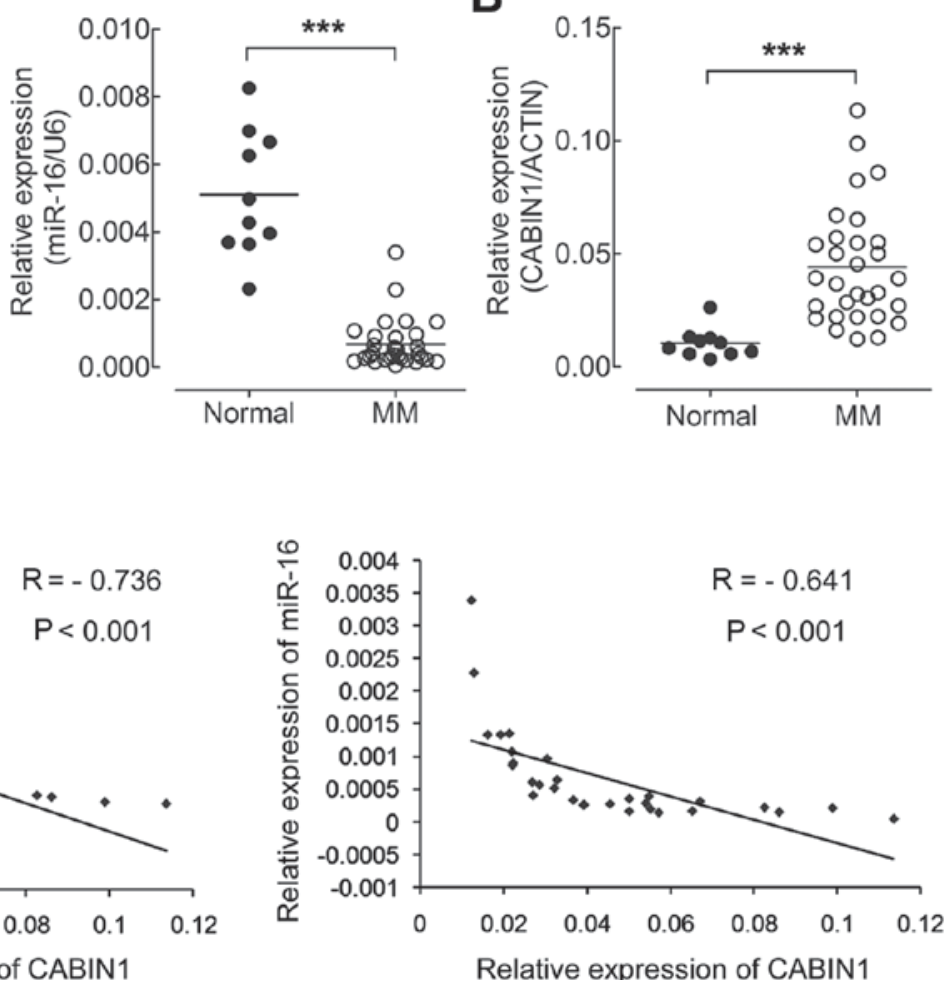

Figure 4. miR-15a and miR-16 downregulate CABIN1 expression and expression of miR-15a and miR-16 are negatively associated with CABIN1 mRNA expression in MM samples. (A) RT-qPCR analysis of miR-15a and miR-16 expression in 30 MM specimens and 10 normal specimens. The expression of miR-15a and miR-16 was normalized to U6 small nuclear RNA. (B) RT-qPCR analysis of CABIN1 expression in the same MM and healthy volunteers as in (A). The expression of CABIN1 was normalized to $\beta$-actin. (C) A negative Spearman's correlation between miR-15a/miR-16 and CABIN1 mRNA levels was observed in $30 \mathrm{MM}$ specimens. ${ }^{* * *} \mathrm{P}<0.01$ by one-way analysis of variance followed by the Student Newman-Keuls test. T, tumor specimens; $\mathrm{N}$, normal specimens; MM, multiple myeloma; miR-15a, microRNA-15a; CANIB1, calcineurin-binding protein 1; RT-qPCR, reverse transcription-quantitative polymerase chain reaction.

$(n=30)$, following which the expression levels of miR-15a and miR-16 were analyzed using RT-qPCR and normalized to an endogenous control (U6 RNA). miR-15a and miR-16levels were significantly decreased in MM specimens compared with in healthy volunteers $(n=10$; Fig. 4A; $P<0.001)$, which was concordant with the results of certain previous studies $(8,19)$. RT-qPCR analysis was performed to detect the mRNA expression of CABIN1 in the same MM specimens and normalized to $\beta$-actin. As presented in Fig. 4B, CABIN1 mRNA levels were significantly increased in MM specimens compared with healthy volunteers. RNA levels of miR-15a and miR-16, and mRNA levels of CABIN1 in MM tissues were analyzed by Pearson's correlation coefficient analysis. CABIN1 mRNA expression levels were negatively correlated with those of miR-15a $(\mathrm{R}=-0.736 ; \mathrm{P}<0.001)$ and $\mathrm{miR}-16(\mathrm{R}=-0.641 ; \mathrm{P}<0.001)$ in MM tissues (Fig. 4C). Collectively, these results suggested that high endogenous miR-15a and miR-16levels may target and downregulate the expression of CABIN1 in MM.

\section{Discussion}

MM is characterized by multi-stage cellular transformation and complex genomic and epigenetic abnormalities (22). The deregulation of miRNAs in cancer often arises following changes to the genome and epigenome (23). Numerous miRNA genes are located at cancer-associated fragile genomic loci that frequently undergo mutations and could represent novel biomarkers $(5,24)$. Several studies suggest that miRNAs regulate MM oncogenesis, providing functional information on the function of specific miRNAs inpatients with MM, characterized by increased oncogenic miRNA expression $(19,25,26)$. Previous studies have reported that miR-15a and miR-16 are downregulated in numerous types of cancer, including MM $(8,19)$. However, the biological relevance and underlying mechanisms of the downregulation of miR-15a and miR-16 in MM are not yet clear.

Expression of miR-15a and miR-16 are decreased or totally absent in MM and are hypothesized to have same target genes owing to their sequence similarity. In order to investigate the biological significance and the underlying mechanisms of the downregulation of miR-15a and miR-16 in MM, previously published CLASH data was screened for potential miR-15a/miR-16 target sites in CABIN1 mRNA.

As the overexpression of CABIN1 increases the proliferation of cancer cells by physically interacting with p53 and repressing the transcriptional activity of p53 (18), and considering that CABIN1 is a direct target of miR-15a and miR-16, it was hypothesized that miR-15a and miR-16 may affect MM cell viability through CABIN1.

To assess this hypothesis, again-of-function study was performed, the results of which indicated that miR-15a and miR-16 directly target CABIN1 mRNA and negatively regulate the expression of CABIN1 at the mRNA and protein level in MM cells. Using a cell proliferation assay, upregulation of miR-15a and miR-16 was revealed to inhibit the proliferation of MM cells by targeting CABIN1. Furthermore, the correlation between miR-15a and miR-16 expression levels 
and the level of CABIN1 mRNA in MM specimens was analyzed. miR-15a and miR-16 expression was significantly lower in MM specimens than in normal specimens, whereas the levels of CABIN1 mRNA were significantly higher in MM specimens than in normal specimens. CABIN1 mRNA levels were negatively correlated with miR-15a $(R=-0.736 ; P<0.001)$ and miR-16 $(\mathrm{R}=-0.641 ; \mathrm{P}<0.001)$ expression levels in $\mathrm{MM}$ tissues. Collectively, the results of the present study indicate that miR-15a and miR-16 are downregulated and negatively associated with CABIN1 mRNA levels in MM specimens.

Future studies must investigate gene expression changes in the p53-p21 signaling pathway, in order to verify that miR-15a miR-16 affects MM cell viability through CABIN1, and that CABIN1 increases the proliferation of cancer cells via suppressing p53 transcriptional activity towards its target genes, including CDKN1A. Other studies have indicated that miR-15 could inhibit protein kinase B, nuclear factor $\kappa$-light-chain-enhancer of activated $B$ cells and vascular endothelial growth factor activity, thus exerting antitumor effects $(8,18)$, which should be considered in future studies.

In summary, the results of the present study suggest that miR-15a and miR-16 may serve as tumor suppressor genes in MM pathogenesis, and that miR-15a and miR-16 may be a promising therapeutic target in the treatment of MM.

\section{References}

1. Palumbo A and Anderson K: Multiple myeloma. N Engl J Med 364: 1046-1060, 2011.

2. Raab MS, Podar K, Breitkreutz I, Richardson PG and Anderson KC: Multiple myeloma. Lancet 374: 324-339, 2009.

3. De Angelis R, Minicozzi P, Sant M, Dal Maso L, Brewster DH, Osca-Gelis G, Visser O, Maynadié M, Marcos-Gragera R, Troussard X, et al: Survival variations by country and age for lymphoid and myeloid malignancies in Europe 2000-2007: Results of EUROCARE-5 population-based study. Eur J Cancer 51: 2254-2268, 2015.

4. Lionetti M, Agnelli L, Mosca L, Fabris S, Andronache A, Todoerti K, Ronchetti D, Deliliers GL and Neri A: Integrative high-resolution microarray analysis of human myeloma cell lines reveals deregulated miRNA expression associated with allelic imbalances and gene expression profiles. Genes Chromosomes Cancer 48: 521-531, 2009.

5. Calin GA, Sevignani C, Dumitru CD, Hyslop T, Noch E, Yendamuri S, Shimizu M, Rattan S, Bullrich F, Negrini $M$ and Croce CM: Human microRNA genes are frequently located at fragile sites and genomic regions involved in cancers. Proc Natl Acad Sci USA 101: 2999-3004, 2004.

6. Chen L, Li C, Zhang R, Gao X, Qu X, Zhao M, Qiao C, Xu J and Li J: MiR-17-92 cluster microRNAs confers tumorigenicity in multiple myeloma. Cancer Lett 309: 62-70, 2011.

7. Gao X, Zhang R, Qu X, Zhao M, Zhang S, Wu H, Jianyong L and Chen L: MiR-15a, miR-16-1 and miR-17-92 cluster expression are linked to poor prognosis in multiple myeloma. Leuk Res 36: 1505-1509, 2012.

8. Sun CY, She XM, Qin Y, Chu ZB, Chen L, Ai LS, Zhang L and $\mathrm{Hu}$ Y: miR-15a and miR-16 affect the angiogenesis of multiple myeloma by targeting VEGF. Carcinogenesis 34: 426-435, 2013.

9. Calin GA, Dumitru CD, Shimizu M, Bichi R, Zupo S, Noch E, Aldler H, Rattan S, Keating M, Rai K, et al: Frequent deletions and down-regulation of micro-RNA genes miR15 and miR16 at 13q14 in chronic lymphocytic leukemia. Proc Natl Acad Sci USA 99: 15524-15529, 2002.
10. Tung YT,Huang PW, Chou YC,LaiCW, Wang HP,HoHC, Yen CC, Tu CY, Tsai TC, Yeh DC, et al: Lung tumorigenesis induced by human vascular endothelial growth factor (hVEGF)-A165 overexpression in transgenic mice and ameliora-tion of tumor formation by miR-16. Oncotarget 6: 10222-10238, 2015.

11. Kang W, Tong JH, Lung RW, Dong Y, Zhao J, Liang Q, Zhang L, Pan Y, Yang W, Pang JC, et al: Targeting of YAP1 by microRNA-15a and microRNA-16-1 exerts tumor suppressor function in gastric adenocarcinoma. Mol Cancer 14: 52, 2015.

12. Huang E, Liu R and Chu Y: miRNA-15a/16: As tumor suppressors and more. Future Oncol 11: 2351-2363, 2015.

13. Hao M, Zhang L, An G, Sui W, Yu Z, Zou D, Xu Y, Chang H and Qiu L: Suppressing miRNA-15a/-16 expression by interleukin-6 enhances drug-resistance in myeloma cells. J Hematol Oncol 4: $37,2011$.

14. Hao M, Zhang L, An G, Meng H, Han Y, Xie Z, Xu Y, Li C, Yu Z, Chang $\mathrm{H}$ and Qiu L: Bone marrow stromal cells protect myeloma cells from bortezomib induced apoptosis by suppressing microRNA-15a expression. Leuk Lymphoma 52: 787-794, 2011.

15. Hideshima T, Mitsiades C, Akiyama M, Hayashi T, Chauhan D, Richardson P, Schlossman R, Podar K, Munshi NC, Mitsiades N and Anderson KC: Molecular mechanisms mediating antimyeloma activity of proteasome inhibitor PS-341. Blood 101: 1530-1534, 2003.

16. Livak KJ and Schmittgen TD: Analysis of relative gene expression data using real-time quantitative PCR and the 2(-Delta Delta C(T)) method. Methods 25: 402-408, 2001.

17. Helwak A, Kudla G, Dudnakova T and Tollervey D: Mapping the human miRNA interactome by CLASH reveals frequent noncanonical binding. Cell 153: 654-665, 2013.

18. Jang H, Choi SY, Cho EJ and Youn HD: Cabin1 restrains p53 activity on chromatin. Nat StructMolBio 16: 910-915, 2009.

19. Roccaro AM, Sacco A, Thompson B, Leleu X, Azab AK, Azab F, Runnels J, Jia X, Ngo HT, Melhem MR, et al: MicroRNAs $15 \mathrm{a}$ and 16 regulate tumor proliferation in multiple myeloma. Blood 113: 6669-6680, 2009.

20. Liu W, Youn HD and Liu JO: Thapsigargin-induced apoptosis involves cabin1-MEF2-mediated induction of Nur77. Eur J Immunol 31: 1757-1764, 2001.

21. Choi SY, Jang H, Roe JS, Kim ST, Cho EJ and Youn HD: Phosphorylation and ubiquitination-dependent degradation of CABIN1 releases p53 for transactivation upon genotoxic stress. Nucleic Acids Res 41: 2180-2190, 2013.

22. Chng WJ, Glebov O, Bergsagel PL and Kuehl WM: Genetic events in the pathogenesis of multiple myeloma. Best Pract Res Clin Haematol 20: 571-596, 2007.

23. Ventura A and Jacks T: MicroRNAs and cancer: Short RNAs go a long way. Cell 136: 586-591, 2009.

24. He L, Thomson JM, Hemann MT, Hernando-Monge E, Mu D, Goodson S, Powers S, Cordon-Cardo C, Lowe SW, Hannon GJ and Hammond SM: A microRNA polycistron as a potential human oncogene. Nature 435: 828-833, 2005.

25. Pichiorri F, Suh SS, Ladetto M, Kuehl M, Palumbo T, Drandi D, Taccioli C, Zanesi N, Alder H, Hagan JP, et al: MicroRNAs regulate critical genes associated with multiplemyeloma pathogenesis. Proc Natl Acad Sci USA 105: 12885-12890, 2008.

26. Misiewicz-Krzeminska I, Sarasquete ME, Quwaider D, Krzeminski P, Ticona FV, Paíno T, Delgado M, Aires A, Ocio EM, García-Sanz R, et al: Restoration of microRNA-214 expression reduces growth of myeloma cells through positive regulation of P53 and inhibition of DNA replication. Haematologica 98: 640-648, 2013. 